LAW RENCE LIVERMORE N A T IO N A L LABORATORY

\section{Inclusion of Scatter in HADES: Final Report}

M. B. Aufderheide

December 21, 2010 
This document was prepared as an account of work sponsored by an agency of the United States government. Neither the United States government nor Lawrence Livermore National Security, LLC, nor any of their employees makes any warranty, expressed or implied, or assumes any legal liability or responsibility for the accuracy, completeness, or usefulness of any information, apparatus, product, or process disclosed, or represents that its use would not infringe privately owned rights. Reference herein to any specific commercial product, process, or service by trade name, trademark, manufacturer, or otherwise does not necessarily constitute or imply its endorsement, recommendation, or favoring by the United States government or Lawrence Livermore National Security, LLC. The views and opinions of authors expressed herein do not necessarily state or reflect those of the United States government or Lawrence Livermore National Security, LLC, and shall not be used for advertising or product endorsement purposes.

This work performed under the auspices of the U.S. Department of Energy by Lawrence Livermore National Laboratory under Contract DE-AC52-07NA27344. 


\section{Inclusion of Scatter in HADES: Final Report}

IAA No. HSHQDC-08-X-00388

DNDO Program Manager: Joel Rynes

Issued: December 20, 2010

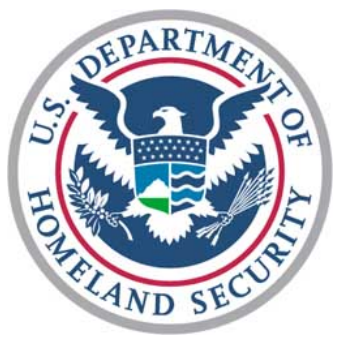

Prepared by: Dr. Maurice B. Aufderheide III

$\frac{\text { aufderheide1@llnl.gov }}{\text { Mail Stop L-096 }}$
7000 East Avenue
Livermore, CA 95550




\section{Overview}

Covert nuclear attack is one of the foremost threats facing the United States and is a primary focus of the War on Terror. The Domestic Nuclear Detection Office (DNDO), within the Department of Homeland Security (DHS), is chartered to develop, and improve domestic systems to detect and interdict smuggling for the illicit use of a nuclear explosive device, fissile material or radiological material.

The CAARS (Cargo Advanced Automated Radiography System) program is a major part of the DHS effort to enhance US security by harnessing cutting-edge technologies to detect radiological and nuclear threats at points of entry to the United States. DNDO has selected vendors to develop complete radiographic systems. It is crucial that the initial design and testing concepts for the systems be validated and compared prior to the substantial efforts to build and deploy prototypes and subsequent large-scale production.

An important aspect of these systems is the scatter which interferes with imaging. Monte Carlo codes, such as MCNP (X-5 Monte Carlo Team, 2005 Revision) allow scatter to be calculatied, but these calculations are very time consuming. It would be useful to have a fast scatter estimation algorithm in a fast ray tracing code. We have been extending the HADES raytracing radiographic simulation code to model vendor systems in a flexible and quick fashion and to use this tool to study a variety of questions involving system performance and the comparative value of surrogates. To enable this work, HADES has been linked to the BRL-CAD library (BRL-CAD Open Source Project, 2010), in order to enable the inclusion of complex CAD geometries in simulations, scanner geometries have been implemented in HADES, and the novel detector responses have been included in HADES.

A major extension of HADES which has been required by this effort is the inclusion of scatter in these radiographic simulations. Ray tracing codes generally do not easily allow the inclusion of scatter, because these codes define a source and a grid of detector pixels and only compute the attenuation along rays between these points. Scatter is an extremely complex set of processes which can involve rays which change directions many times between the source and detector. Scatter from outside the field of view of the imaging system, as well as within the field of view, can have an important role in image formation. In this report, we will describe how we implemented a treatment of scatter in HADES. We begin with a discussion of how we define scatter in Section 2, followed by a description of how single Compton scatter is now included in HADES in Section 3. In Section 4 we report a set of verification tests against MCNP and tests of how the technique scales with image size, number of scatters allowed and number of processors used in the calculations. In Section 5, we describe how we plan to extend this approach to other forms of scatter and conclude in Section 6. It should be emphasized that the purpose of this report is to show that a form of scatter has been implemented in HADES and has been verified against MCNP. Validation, the process of comparing simulation and experiment, is a future task.

\section{What is X-Ray Scatter?}

Transmission X-Ray radiography has been used in Physics and Engineering for over a century. At its simplest, a source of photons in the X-ray or Gamma Ray range is shined through an object and the transmitted radiation forms a shadow of the object which has been illuminated. This process allows the researcher to inspect an object without touching it and, in the case of tomography, infer a reconstruction of the linear absorption coefficient of the object. 
The image of the object recorded in transmission radiography is composed of photons in two components: direct radiation, which has not been scattered or absorbed by the object, and scattered radiation, which has been produced either within the object or system in some way as a result of the incident illumination. HADES, as a ray-tracing code, had, until recently, focused on the direct radiation, since it forms the image of interest in transmission radiography. "Scatter" actually has many sources and requires further delineation before one decides how it could be included in a ray-tracing code. In this document, "scatter" will include any physical process which results in the production of photons which did not originate in the source. In this discussionof scatter processes, we draw on two discussions by Evans (Evans, 1985) and Rybicki and Lightman (Rybicki \& Lightman, 1979).

The first form of X-Ray scatter which comes to mind is Compton scatter, in which a photon scatters from an electron in the system illuminated by the source beam. The photon is scattered into a new direction and new energy and the electron is sent off in another direction with energy in the $\mathrm{MeV}$ range. As a photon moves through an object, it could experience several Compton scatters, further scrambling its energy and direction. Also, with each Compton scatter, an electron can be freed from the atom. The differential Compton scatter formula is given by the Klein-Nishina expression (Klein \& Nishina, 1929) for scattering from free electrons. It must be remembered that, for the objects of interest here, the electrons are initially bound, which results in the Compton cross section tapering to 0 as the incident photon energy declines below 100 $\mathrm{keV}$. The Klein-Nishina cross section does not include these atomic effects.

If the scattered electrons in the $\mathrm{MeV}$ energy range are introduced into a material, they are quickly decelerated as a result of multiple Coulomb scatters from other electrons and nuclei. In the process of decelerating, they can emit radiation, known as bremsstrahlung. The radiation produced from bremsstrahlung is a continuous photon energy whose highest, or endpoint, energy is given by the incident energy of the electron. In the systems of interest here, the electron which results from Compton scattering can then emit this radiation, here called secondary bremsstrahlung because this process is the result of a Compton scatter. In our work, we do consider secondary bremsstrahlung to be a form of X-Ray scatter, since it is a result of the initial illumination of the system by the X-Ray source.

Compton scatter is often called "incoherent" scatter, because the incident photon does not see the full cloud of electrons around each nucleus, but rather sees the individual electrons around each nucleus. For coherent scatter (also known as elastic or Rayleigh scatter), the incident electron sees and scatters off each atom's full electron cloud. Such scatter is strongly forward peaked and does not change the energy of the photon (energy and momentum conservation being provided by the massive nucleus). The cross section for coherent scatter is typically weaker than any of the other processes described here. Because of this weakness, the extreme forward peaking and the lack of change to the photon energy, we have generally neglected Rayleigh scattering. However, there are some indications that, for heavily shielded situations at intermediate energies (in the $1 \mathrm{MeV}$ range or lower), Rayleigh scattering could be an important background effect. The forward peaking of this process could lead to a blurring of the direct image much larger than can be accounted for by processes in the detection medium itself. This effect is not expected to be seen in the scanners of interest here. However, the infrastructure developed below could also be applied to this process, if the cross sections for coherent scatter were included in the HADES scatter treatment.

Another process which is important at these energies is pair production. In this process, an electron and positron are produced when a photon of sufficient energy scatters off the 
Coulomb field of the nuclei in the system. Some fraction of the incident photon energy is dumped into the production of this pair. As a result of this process the incident photon has definitely lost energy and has likely changed direction, which makes this a scattering process by our definition. In addition, a new electron and positron have been introduced into the system, allowing secondary bremsstrahlung to occur (albeit generally at lower energies than in the case of the Compton electron), generating more scattered radiation in the system. In addition, the positron will eventually annihilate with an electron in the system, generating $511 \mathrm{keV}$ emission lines, another source of "scattered" radiation in the system.

Photoelectric absorption is a process which is important in calculating the attenuation of these systems, but it does not fit our definition of a scattering process. In this process, the incident photon is completely absorbed, resulting in a bound electron in the system either escaping from its atom, or being shifted to a bound state of higher energy. Since no photons result from this immediate process, photoelectric absorption is not a scatter source by our definition. It is possible for the electron hole to be filled by an electron dropping from a higher orbital, emitting typically a low energy X-Ray in the process. This situation is known as Auger emission, but it is generally not relevant when we are dealing with photon energies in the $\mathrm{MeV}$ energy range.

At higher energies (greater than roughly $10 \mathrm{MeV}$ ), other scatter processes occur. We will only mention them here, but they are generally not relevant because our sources are not energetic enough for these processes to occur often. One example is triplet production (Hubbell, 2006), in which the electron-positron pair is produced in the electric field of an electron. A "triplet" of tracks is produced, corresponding to two electrons and one positron Another process is Delbrück scattering (McDonald, 1998), in which the photon scatters off the Coulomb field of nuclei in the system. Yet another process is nuclear fluorescence (Schiff, 1946), in which the photon is absorbed by a nucleus, putting it in an excited state, from which it decays emitting a cascade of $\mathrm{MeV}$ scale photons.

Lastly, it is possible for the photon to excite the giant dipole resonance of a nucleus (Baldwin \& Klaiber, 1947) (Wattenberg, 1947). If this resonance (or portions of its tail) is above the neutron emission threshold of the nucleus, a spray of scattered neutrons can result from the incident photon beam. These neutrons can then reach detectors and provide spurious signals for the detectors, which were seeking to detect photons. This process is not technically scatter by the definition given above, since photons are not emitted. Nevertheless, it is an important process and can affect detectors. While this process is beyond the scope of this effort, one could imagine using the single scatter infrastructure in HADES reported here to model this effect, using photo-neutron production cross sections.

\section{Strategy for Single Compton Scatter Inclusion in HADES}

\section{Overview}

Most of these processes discussed in Section 2 are computed in full featured Monte Carlo codes (with the exception of the higher energy processes), but at considerable computational cost. To attempt to put all of these processes into HADES at once was deemed to be too ambitious a goal. Indeed, it was not clear how to even approximate the processes which involved multiple steps and/or the transport of electrons in a ray tracing code such as HADES. It 
was judged that an incremental approach had a higher probability of producing a tool of practical use in a reasonable amount of time.

When one does scatter simulations with a full Monte Carlo treatment, it can be seen that the term from single Compton scatters has the largest individual contribution, particularly in transmission geometries (this may not be true for backscatter geometries). Thus there would be some value in beginning the effort by only treating single Compton scatter. Happily, this process is relatively simple from a computational standpoint.

Initially, HADES was built on a transmission radiographic paradigm in which there is only one point source, which defines the start point of either a fan beam or cone beam geometry (HADES also could also simulate parallel beams as a special case). However, even in transmission radiography, sources are not points and have finite spot size. In addition, there is a rich variety of diagnostic techniques in which imaging is done either with or of an extended source. Examples include pinhole imaging (Dicke, 1968) (Groh, Hayat, \& Stroke, 1972), (Holt, 1976), backlighting imaging (Key, Lewis, Lunney, Moore, Hall, \& Evans, 1978) (Miyanaga, Kato, \& Yamanaka, 1983), and transmission radiography of radioactive objects (Moses, Gayshan, \& Gektin, 2006). In order to study these situations, HADES had been extended to model multiple sources in the same simulation.

For such an "extended source", HADES sets up an ensemble of "sourcelets", with a given spatial weighting over the ensemble. For each sourcelet, HADES does a complete radiographic simulation. Each simulation is then added to the final radiograph in intensity units, given the spatial weighting for each sourcelet. This addition of images was initially challenging because HADES originally only computed pathlengths through objects and not the transmitted intensity (pathlength is the negative natural log of the transmitted intensity for a monochromatic source; for a monochromatic problem, pathlength can be added without keeping track of the order in which objects intersect the beam). As part of the effort to enable the treatment of extended sources, HADES transitioned to operating in units of transmitted flux.

This paradigm shift was completed before the current project began but was crucial for two reasons. First, it puts HADES on a much more physical footing, since transmitted fluxes are quantities which are much more relevant for physics-based modeling of detectors and scattering. Second, this new approach allowed HADES to treat extended sources in highly parallelized way. Parallelization allows these more computationally intensive problems to be computed in reasonable wall clock times.

The extension to single scatter then became a realizable task, since scatter points within objects could be seen as additional sourcelets, with their weighting governed by the incident attenuated flux, Klein-Nishina differential cross section, attenuation between the sourcelet and the detector and the solid

Figure 1: Notional approach to including single Compton scatter in HADES. 
angle of the detector. A schematic of the approach devised for HADES is given in Figure 1. The sourcelet segments are delineated in the image along one of the rays and one sourcelet is shown emitting scattered rays. Note that this image assumes that a scanner geometry is being used, so that scatter only occurs in the plane of the detector. However, as implemented in HADES the approach works in either the plane or into a full area imaging array. The figure also demonstrates another feature of the current implementation. At present, HADES only computes scatter from a BRL-CAD object, not meshes or traditional HADES solid-body objects.

Eventually, scatter will be extended to meshes, but there is no plan to extend treatment to solidbody objects because BRL-CAD has better coverage of such objects.

\section{Implementation}

Let $n$ be the number of forced Compton scatters per Compton path length. This is a userset parameter.

Before ray tracing begins, all cross sections as a function of energy for the calculation are assembled from the Evaluated Photon Data Library (Cullen, Perkins, \& Rathkopf, 1990). This library includes 5 processes: photoelectric absorption, coherent scattering, Compton scatter, pair production and triplet production all integrated over angle (in HADES, we do not include coherent scatter because it is expected to scatter back into the beam, although as discussed above this may be a bad assumption). The first HADES revision for this project involved separating out the Compton scatter cross section and finding its maximum value for each material. We seek the maximum value, $\sigma^{\mathrm{Max}}$ Compton for estimating the number of Compton scatter lengths through objects later in the calculation.

For each source to detector ray in the problem, the following operations are performed:

a) A complete history of all intersections of the ray with objects is compiled as an ordered list of entrance and exit points (and their distance $\Delta t$ ) and materials/densities traversed.

b) This list is expanded into a linked list of $n^{*}$ the number of Compton lengths in each material segment traversed. A Compton length is defined as $1 /\left[\rho \mathrm{N}_{\mathrm{A}} \sigma^{\mathrm{Max}}{ }_{\text {Compton }}\right]$, so that the number of Compton scatter sourcelets in a segment is $\Delta t n /\left[\rho \mathrm{N}_{\mathrm{A}} \sigma^{\operatorname{Max}}{ }_{\text {Compton }}\right]$. If this quantity is less than one, it is set to one, to ensure at least one Compton scatter in the object. For each element of this new linked list, the center of each subsegment, material composition and density are saved.

c) Each of these subsegments becomes a Compton scatter sourcelet. For each of these sourcelets, do the following:

i) Compute the energy dependent attenuation of the incident spectrum $\mathrm{S}\left(\mathrm{E}_{\mathrm{bin}}\right)$ to this point (integrating over all subsegments between the source and this sourcelet). Call this attenuated spectrum $\mathrm{S}^{\prime}$ ( $\mathrm{E}_{\mathrm{bin}}$,sourcelet). Compute the energy dependent pathlength from the sourcelet to the detector array. Call this attenuation $\exp \left(-\tau\left(\mathrm{E}_{\text {bin }}{ }^{\text {sourcelet,pixel })}\right)\right.$.

ii) For each energy bin of the spectrum:

(1) For each detector pixel:

(a) Compute $\mathrm{d} \sigma / \mathrm{d} \Omega\left(\mathrm{E}_{\mathrm{bin}}, \theta^{\prime}\right)$, using the angle $\theta^{\prime}$ between the incident ray and the ray pointing from the sourcelet to the detector pixel. Note that 
$\mathrm{d} \sigma / \mathrm{d} \Omega\left(\right.$ Ebin, $\left.\theta^{\prime}\right)$ is the Klein-Nishina expression, normalized by the total Compton cross section from the Evaluated Photon Data Library. This is done to kill off the Klein-Nishina expression for low incident photon energy. At low energies, atomic effects, not included in Klein Nishina, dominate the actual cross section. This normalization is not strictly correct, but is an approximation used in HADES for this effort.

(b) Compute the solid angle, $\mathrm{d} \Omega$ (pixel), for the detector pixel as seen from this sourcelet. It can be shown that the solid angle of the pixel is the surface area of the pixel divided by the square of the distance from the sourcelet to the pixel. Also, compute the cosine of the angle between the sourcelet to detector ray and the detector normal. The detector normal here is assumed to be the ray from the detector to the source (that is, the detector is always assumed to be pointing at the source). This is an assumption which will be revised in the future.

(c) Determine the energy bin E' bin into which the Compton scatter will go for incident photons in this energy bin scattering to this detector pixel.

$$
E_{b i n}^{\prime}=\frac{E_{b i n}}{1+\frac{E_{b i n}}{m_{e} c^{2}}\left(1-\cos \theta^{\prime}\right)}
$$

All of the energy from the incident energy bin is scattered into this bin. There is no calculation of overlap of the scattered bin with neighboring bins. This is another approximation which could be improved upon in future work.

(d) Finally, everything is assembled into a special scatter structure, which is exactly like a HADES image structure (a stack of images, one for each energy bin):

$\operatorname{Scatter}\left(E_{b i n}^{\prime}, s, p\right)=S^{\prime}\left(E_{b i n}, s\right) \times \rho(s) \times \delta \ell(s) \times \frac{d \sigma}{d \Omega}\left(E, \theta^{\prime}\right) \times \frac{d A}{R^{2}} \times \cos (\beta) \times \exp \left(-\tau\left(E_{b i n}^{\prime}, s, p\right)\right)$ where s refers to sourcelet, $\mathrm{p}$ refers to detector pixel, $\rho$ and $\delta \ell$ are the density and length of the soucelet zone respectively, $\mathrm{dA}$ is the area of the detector, $\mathrm{R}$ is the distance from the sourcelet center to the detector center, and $\beta$ is the angle between the detector normal and the sourcelet-pixel ray.

After all of these loops over detector pixel, source energy bin, sourcelets, and incident rays are completed, the total scatter is stored in a separate scatter structure which can be added to the direct radiation structure and then post processed using standard HADES blurring and noise modeling. Presently, HADES prints out this scatter function in a separate image, processed just as a direct image is processed. We do not yet add the direct and scatter signals because we are still testing scatter computation. It is also possible for the user to pick a detector pixel and list the spectral content of the scatter at this pixel. We use this capability in the verification tests described below.

A final comment should be made about the numerical cost of these calculations. For an image of size $\mathrm{NxN}$, the time for a simple HADES calculation scales as $\mathrm{N}^{2}$. For a HADES 
calculation of a scanner including scatter, the calculation scales as $\mathrm{N}^{3}$. This $\mathrm{N}^{3}$ scaling can be assuaged by the use of threading and massively parallel computations. For a HADES calculation including scatter onto a standard area array, the calculation scales as $\mathrm{N}^{4}$. This severe scaling can be partly relieved by the use of threading and massively parallel computations, but very quickly the $\mathrm{N}^{4}$ scaling beats the increased availability of processors. One strategy for beating this scaling in the future might be to only compute scatter at $\sqrt{ } \mathrm{N}$ points in each direction. The full scatter array could then be filled out by use of interpolation operations between the $\sqrt{ } \mathrm{Nx} \sqrt{ } \mathrm{N}$ locations where scatter would be computed. In such a strategy, the scaling would again be $\mathrm{N}^{3}$, which could be handled by large numbers of CPUs in the calculation. Such an approach would be fairly accurate because scatter generally has a low spatial frequency at the detector plane.

\section{Verification Tests and Physics Implications}

\section{Verification tests}

In order to verify that the above HADES single scatter computation is correct, we have crafted a test case which allows comparison between MCNP and HADES. The case is illustrated in Figure 2 at right. The cyan cube is a CAARS cube composed of tungsten at density 19.3 $\mathrm{g} / \mathrm{cc}$. The blue cube is piece of a shielding wall, designed to be $7.63 \mathrm{~cm}$ thick and $(8 \mathrm{~cm})^{2}$ wide. We

Figure 2: Layout for verification test. Source is red sphere, Detector plane is yellow square and the three detector points of interest are green spheres.
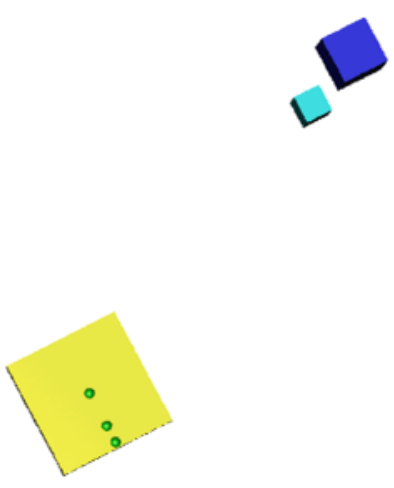
have selected these dimensions so that the cube and wall contribute comparable amounts of attenuation and Compton scatter. We have selected a relatively thin wall because greater thickness simply slows down the time for convergence of the Monte Carlo calculation, making verification more difficult.

The source is $304.5 \mathrm{~cm}$ from the center of the wall, $348.84 \mathrm{~cm}$ from the center of the cube and $600 \mathrm{~cm}$ from the detector plan. The detector points are at $0 \mathrm{~cm}, 6 \mathrm{~cm}$ and $9 \mathrm{~cm}$ from the radiographic axis. These numbers were chosen because they

correspond to the center of the cube/wall shadow, the center of the wall shadow outside the cube and outside the shadow of both objects. These objects are illuminated with two sources: $6 \mathrm{MeV}$ and $9 \mathrm{MeV}$. These sources are not spectra, but rather monochromatic sources. These definitions were chosen because they allow all radiation of lower energy to be definitely classified as the result of some kind of scatter process as defined above, which is extremely useful for verification studies. These two energy values were chosen because they correspond to the common endpoint energies used in modern scanners and are thus extremal tests of such systems. In HADES, a special spectrum file is used in each case. $100 \mathrm{keV}$ bins are used, starting at $50 \mathrm{keV}$ and extending $50 \mathrm{keV}$ above the endpoint energy of interest. For $6 \mathrm{MeV}$, this corresponds to a 60 bin spectrum, will all 0 values until the $5.95 \mathrm{MeV}$ to $6.05 \mathrm{MeV}$ bin, which has a strength of 1 . For 9 $\mathrm{MeV}$, this corresponds to a 90 bin spectrum, will all 0 values until the $8.95 \mathrm{MeV}$ to $9.05 \mathrm{MeV}$ bin, which has a strength of 1. In MCNP, the sources are defined as point sources with fixed 
energy. The binning of the detector tallies are chosen so that the bins match those used in HADES. "f5" detectors are used in MCNP, which tallies x-ray flux at the specified point.

We have modeled this system with HADES and with MCNP 6.1.42. We have done the Monte Carlo simulations in three ways. First, we have run with full electron transport and all scatter processes on, except for coherent scatter. We have neglected coherent scatter because it drastically slows Monte Carlo convergence, HADES does not include it and it is small, relative to the other scatter processes. Second, we have run MCNP with all scatter processes off, except for Compton scatter and we have tallied by numbers of scatters which reach the detector. Third, we have repeated the full scatter case, but with all material densities set to $0.001 \mathrm{~g} / \mathrm{cc}$. This allows us to determine what the flux without any attenuation at all three detectors would be.

In Figure 3, we show the HADES images for a $6 \mathrm{MeV}$ source. The shadows of the cube (inner) and shielding plate (outer) can be seen in the direct image.

The scatter image is strongest in the center and weakens as we move outward; however, this trend is actually only a $3 \%$ drop from center to corner in the image. In Figure 4, we compare the relative strengths of the direct and scattered signals along the central line

of the images. It can be seen that the scattered radiation is weaker than the direct radiation in this case.

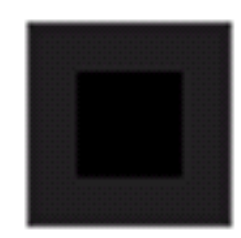

Figure 3: HADES simulated radiographs of direct radiation (left) and for scattered radiation (right). Units are intensity, normalized to 1 .

The scattered radiation at the center of the image is only $9 \%$ of the direct radiation. Similar results are seen for the $9 \mathrm{MeV}$ HADES simulation. In this case, the scattered radiation varies $4 \%$ over the image. The scattered radiation is $17 \%$ of the direct radiation for the $9 \mathrm{MeV}$ source.

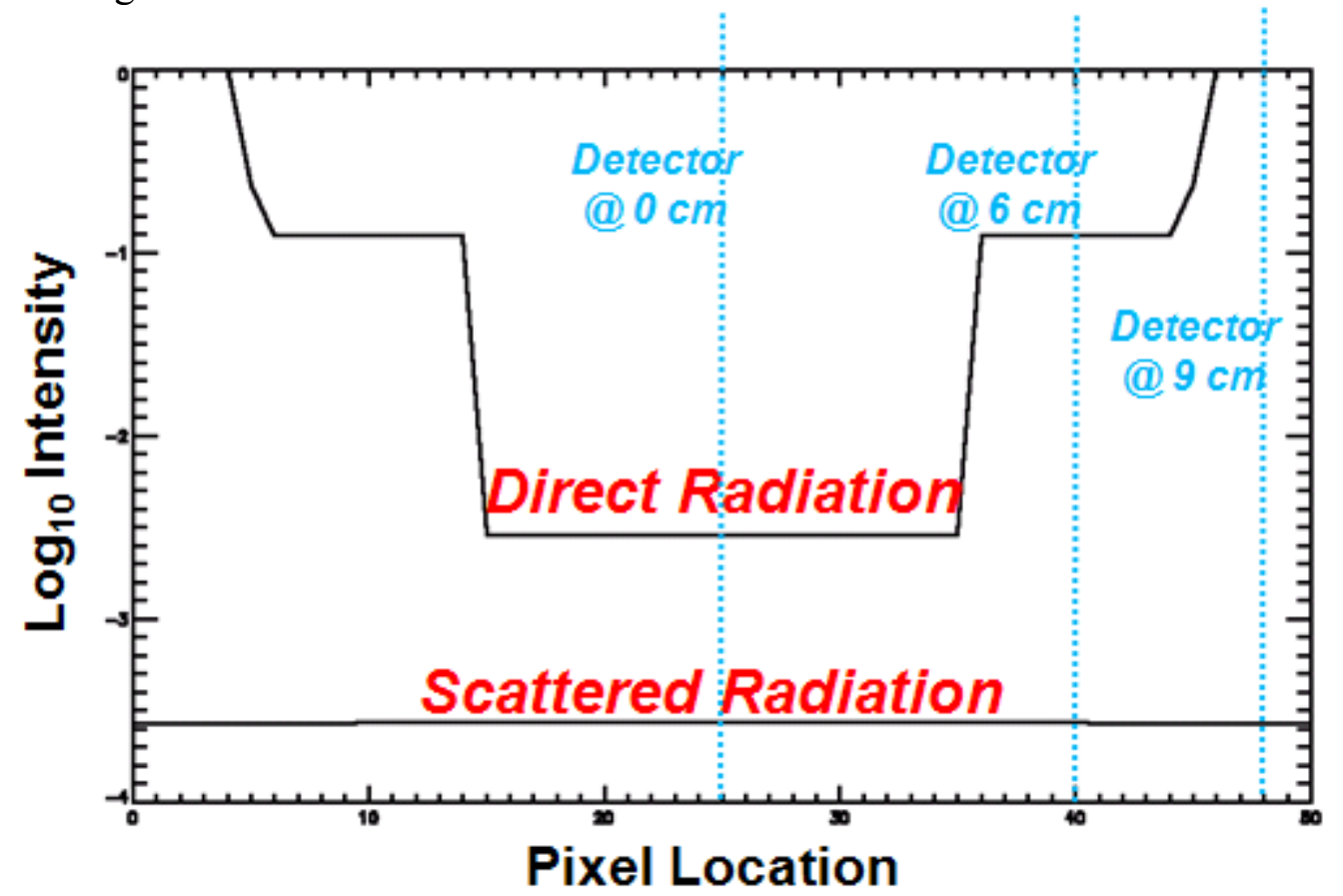

Figure 4: Comparison of direct and scattered radiation across the center of the objects as calculated by HADES.

In Table 1, we show HADES' detailed spectral output of the scatter at each detector. The direct signal is also listed for comparison. For both energies, the detector at $6 \mathrm{~cm}$ sees much more direct signal than the central detector. This result is not surprising, since the central 
detector is obscured by the wall and the CAARS cube. The comparison of the direct radiation between the $6 \mathrm{MeV}$ and $9 \mathrm{MeV}$ cases is more interesting. Generally, X-ray analysts expect materials to become more transparent as photon energy increases. This trend is seen for the $6 \mathrm{~cm}$ detector (a $6 \%$ increase in transmitted intensity from $6 \mathrm{MeV}$ to $9 \mathrm{MeV}$ ), but not the central detector (a $27 \%$ decrease in transmitted intensity from $6 \mathrm{MeV}$ to $9 \mathrm{MeV}$ ). This latter trend can be understood with a more detailed understanding of photon absorption cross sections. For energies below $2 \mathrm{MeV}$ to $5 \mathrm{MeV}$, photon absorption is dominated by the photoelectric cross section, which drops precipitously with increasing photon energy. However, as photon energy increases beyond this range, absorption is dominated by pair production, which increases with energy. The intermediate "valley" also has a strong contribution from Compton scatter, but this mainly fills in the valley somewhat. The exact energy at which the photon absorption cross section stops decreasing with energy varies with the $\mathrm{Z}$ of the material. For steel, this point is at 9 $\mathrm{MeV}$, whereas for Tungsten, this point is $3.78 \mathrm{MeV}$. Thus, for the $6 \mathrm{~cm}$ detector, the steel wall is still on the decreasing absorption with increasing photon energy trend, leading to a more transparent (but only by 0.03 pathlengths) wall as energy increases. For the Tungsten cube however, the trend has turned and the cube's pathlength increases by roughly 0.45 pathlengths. Thus the detector at the center sees a 27\% decrease in transmitted intensity.

\begin{tabular}{|c|c|c|c|c|c|c|c|}
\hline $\begin{array}{c}\text { Energy } \\
(\mathrm{MeV})\end{array}$ & $0 \mathrm{~cm}$ & $6 \mathrm{~cm}$ & $9 \mathrm{~cm}$ & $\begin{array}{c}\text { Energy } \\
(\mathrm{MeV})\end{array}$ & $0 \mathrm{~cm}$ & $6 \mathrm{~cm}$ & $9 \mathrm{~cm}$ \\
\hline 5.7 & - & - & - & 8.7 & - & - & $2.89 \mathrm{e}-5$ \\
\hline 5.8 & - & - & - & 8.8 & - & $6.05 \mathrm{e}-5$ & $1.18 \mathrm{e}-4$ \\
\hline 5.9 & - & $8.76 \mathrm{e}-5$ & $1.30 \mathrm{e}-4$ & 8.9 & $1.79 \mathrm{e}-4$ & $1.64 \mathrm{e}-4$ & $1.12 \mathrm{e}-4$ \\
\hline 6.0 & $2.73 \mathrm{e}-4$ & $1.84 \mathrm{e}-4$ & $1.39 \mathrm{e}-4$ & 9.0 & $1.95 \mathrm{e}-4$ & $1.47 \mathrm{e}-4$ & $1.08 \mathrm{e}-4$ \\
\hline Direct & $2.90 \mathrm{e}-3$ & $1.25 \mathrm{e}-1$ & 1.0 & Direct & $2.12 \mathrm{e}-3$ & $1.31 \mathrm{e}-1$ & 1.0 \\
\hline
\end{tabular}

Table 1: Comparison of scattered spectrum with direct radiation for $6 \mathrm{MeV}$ and $9 \mathrm{MeV}$ sources

The scattered spectrum is equally interesting. In this case the observed scattered intensities are mainly dominated by the possible angles involved in scattering into each detector. Recall that, for a scatter into an angle $\theta$ from the incident ray, the scattered energy $E$ ' is given by

$$
E^{\prime}=\frac{E}{1+\frac{E}{m_{e} c^{2}}(1-\cos \theta)}
$$

where $m_{e}$ is the electron mass. To determine how many energy bins should have been populated, we can look over the objects and seek the scatter point which would yield the largest possible angle between any source ray and the ray to the detector.

Consider the central detector. In this case, the largest possible angle would be from the source to a corner of the wall. For the geometry, that angle is roughly $2.2^{\circ}$. Thus, E' in this case would be $5.95 \mathrm{MeV}$ for the $6 \mathrm{MeV}$ source and $8.89 \mathrm{Mev}$ for the $9 \mathrm{MeV}$ source. Thus we expect only the top bin to be populated by scatter for the central detector in the $6 \mathrm{MeV}$ source and the top two bins for the $9 \mathrm{MeV}$ source. Let us now consider the $9 \mathrm{~cm}$ detector. In this case nearly the largest scatter angle would be a scatter from the negative edge of the wall across to the $9 \mathrm{~cm}$ detector, which corresponds to a $3.2^{\circ}$ angle. For the $6 \mathrm{MeV}$ source this leads to a minimal E' of $5.89 \mathrm{MeV}$ and we see that the upper two bins of the spectrum are populated by HADES for this case. For the $9 \mathrm{MeV}$ source, the minimal E' is $8.76 \mathrm{MeV}$, which result in HADES populating the upper three energy bins for scatter. However, HADES actually sees a small contribution in the fourth energy bin also. This contribution is coming from scatters off the far corners of the wall. 
These points have a slightly larger angle than $3.2^{\circ}$ (actually $3.6^{\circ}$, if the $3 \mathrm{D}$ geometry is worked out) and account for the smaller population of this additional bin.

In Table 2 we display the timings required for a variety of HADES scatter simulations. The scaling of calculation time with various user-set parameters can be seen. We find that the calculation time scales nearly exactly as N4, as seen in comparing Cases 1 and 3 and 2 and 4.

\begin{tabular}{|c|c|c|c|c|}
\hline Case & $\begin{array}{c}\mathrm{E}_{\text {source }} \\
(\mathrm{MeV})\end{array}$ & $\begin{array}{c}\text { Image } \\
\text { Size }\end{array}$ & $\begin{array}{c}\text { \# Sourcelets per } \\
\text { Compton Length }\end{array}$ & $\begin{array}{c}\text { Total CPU Time } \\
(\text { seconds })\end{array}$ \\
\hline 1 & 6 & $(51)^{2}$ & 1 & 1,590 \\
\hline 2 & 9 & $(51)^{2}$ & 1 & 3,000 \\
\hline 3 & 6 & $(101)^{2}$ & 1 & 24,400 \\
\hline 4 & 9 & $(101)^{2}$ & 1 & 44,000 \\
\hline 5 & 9 & $(51)^{2}$ & 10 & 31,000 \\
\hline
\end{tabular}

Table 2: Scaling properties of HADES simulations

Cases 2 and 5 study the scaling of CPU time with the number of sourcelets used per Compton length, $n$. It can be seen that execution time scales linearly with $n$, as one would expect by looking at the algorithm described above. Cases 1 and 2 and 3 and 4 agree that increasing the spectrum size from 61 energy bins to 91 energy bins leads to an increase of CPU time by a factor of roughly 1.8. This is a steeper scaling that linear, which would imply a factor of 1.5 . It is not presently clear why this scaling is not linear. Perhaps the extra scaling comes from the overall overhead of treating larger stacks of images in the case of larger spectra. This difference in scaling could also be the result of the extra energy bins into which scattering can occur for higher energy sources, as seen in Table 1.

All of these results indicate the internal consistency of these HADES simulations, but they need to be verified against the MCNP calculations. We have used two MCNP calculations for each case to compile Table 3, a chart analogous to Table 1. First, we run MCNP with all scatter-producing processes off, except for Compton scatter. Using the FU and FT supplements to the F5 tally, we get subtallies of 1, 2, 3, 4 and 6-10 Compton scatters. All of these runs were performed serially and generated on the order of $1.2 \times 10^{12}$ particles in 21 hours of CPU time. It was found that this was the number of particles needed to generate less than $3-5 \%$ relative error in the tally outputs. However, this relative error had great variation. In particular, for the tallies of multiple Compton scatter, the relative errors became quite large for the higher energy part of the spectrum. At low energies, relative errors were $1-2 \%$, but at higher energies, relative errors increased with energy, up to $50 \%$ or higher. However, these large errors were not particularly relevant because they pertain to a rapidly decreasing high energy tail of these distributions which have no practical relevance.

It is interesting to compare the run times for the HADES simulations and the Monte Carlo runs. The longest HADES run took just over 12 hours, while all of the Monte Carlo runs had satisfactory convergence after 21 hours of CPU time. However, the MCNP runs only computed spectra at 3 detectors, while HADES effectively was computing scatter spectra for $(101)^{2}$ detectors. These results indicate that HADES has the potential to compute scatter much more efficiently than Monte Carlo, given enough physics information. 


\begin{tabular}{|c|c|c|c|c|c|c|c|}
\hline $\begin{array}{c}\text { Energy } \\
(\mathrm{MeV})\end{array}$ & $0 \mathrm{~cm}$ & $6 \mathrm{~cm}$ & $9 \mathrm{~cm}$ & $\begin{array}{c}\text { Energy } \\
(\mathrm{MeV})\end{array}$ & $0 \mathrm{~cm}$ & $6 \mathrm{~cm}$ & $9 \mathrm{~cm}$ \\
\hline 5.7 & - & - & - & 8.7 & - & - & $1.49 \mathrm{E}-05$ \\
\hline 5.8 & - & - & - & 8.8 & - & $3.54 \mathrm{E}-05$ & $4.85 \mathrm{E}-05$ \\
\hline 5.9 & - & $5.82 \mathrm{E}-05$ & $8.70 \mathrm{E}-05$ & 8.9 & $1.66 \mathrm{E}-04$ & $9.83 \mathrm{E}-05$ & $9.94 \mathrm{E}-05$ \\
\hline$* * 6.0 * *$ & $2.92 \mathrm{E}-04$ & $9.49 \mathrm{E}-05$ & $3.05 \mathrm{E}-04$ & $* * 9.0 * *$ & $1.33 \mathrm{E}-04$ & $2.58 \mathrm{E}-05$ & $2.43 \mathrm{E}-04$ \\
\hline$* *$ Total $* *$ & $3.19 \mathrm{E}-03$ & $1.25 \mathrm{E}-01$ & $1.00 \mathrm{E}+00$ & $* *$ Total** & $2.25 \mathrm{E}-03$ & $1.31 \mathrm{E}-01$ & $1.00 \mathrm{E}+00$ \\
\hline
\end{tabular}

Table 3: Comparison of scattered spectrum with direct radiation for $6 \mathrm{MeV}$ and $9 \mathrm{MeV}$ sources. The asterisked rows require special discussion because of how MCNP tallies single Compton scatter. See text for discussion.

For Table 3, we only use the single Compton tally as computed by MCNP. The resulting tally is in units of flux per incident photon. We normalize these results by using the MCNP run with all densities set to $0.001 \mathrm{~g} / \mathrm{cc}$. This provides $\mathrm{I}_{\mathrm{o}}$ in flux per incident photon. This flux is identical to within $0.01 \%$ for all three detectors. Using this normalization, we produce Table 3 which is in the same units as Table $1: \mathrm{I} / \mathrm{I}_{0}$.

It is difficult to compare Monte Carlo results with the HADES results for a number of reasons. First, MCNP includes what HADES would call the "direct", that is unscattered, signal with the single Compton signal. In Table 3 the "**Total**" row gives the raw MCNP result for the highest energy bin. Comparison of this row with the "Direct" row of Table 1 confirms this conclusion. In Table 3 we have attempted to extract the single Compton portion in the "**6.0**" and "**9.0**" row by subtracting the HADES direct estimate from the MCNP total. It can be seen that the agreement between these estimates and the HADES single Compton values agree fairly well for the $0 \mathrm{~cm}$ detector, but agree more poorly as the detector departs from the center line. The main reason for this divergence is the fact that scatter is becoming a smaller and smaller proportion of the total converged MCNP value. Eventually, by the $9 \mathrm{~cm}$ detector, the inferred MCPN value is such a small proportion of the total signal that it is dominated by Monte Carlo noise. Thus, the agreement for the top energy bin of the $0 \mathrm{~cm}$ detector is heartening and $6 \mathrm{~cm}$ and $9 \mathrm{~cm}$ detectors are inconclusive for comparison of this bin.

For the lower energy bins, the HADES calculations are generally roughly $10 \%$ to $40 \%$ larger than the MCNP results. This trend is likely from the HADES approximations which assumed all detector pixels to be facing the source. In actuality, for this planar detector, the normal is in the $-\mathrm{y}$ direction. The effect of this approximation is that the angle between the scattered ray and the detector normal, $\beta$, is closer to 0 in the HADES simulations than it is in the MCNP runs. Since $\beta$ is smaller, $\cos \beta$ is larger, making the HADES values overestimates. Also, this effect should become worse as the detector deviates from the central axis. This trend can also be seen when comparing the values for the lowest three bins of the $9 \mathrm{~cm}$ detector in Table 1 and Table 3.

Another HADES approximation which can make comparisons difficult is the fact that all of the scatter from an energy bin is placed in whichever bin the scattered energy (based on the center value of the initial energy bin) falls. In reality, this scatter should be distributed over all the energy bins over which the starting bin has been stretched as a result of the scatter. For the initial implementation in HADES this has not been done. This approximation can lead to some misclassification of scatter in HADES. Originally, it was thought that this effect would balance out over the many energy bins in a problem. Such a conjecture may be true in general, but in the verification test here, the number of bins involved is small enough that a few misclassification 
errors has made comparison with MCNP difficult. This approximation will be removed in future work.

Another aspect which may make comparison difficult is the fact that we ran MCNP with full physics on for Compton scattering. It is possible to run MCNP in a "Simple Physics" mode, in which the Klein-Nishina cross section is used, instead of the full atomic form factors. In future work, we will investigate whether this option allows a more transparent comparison with the HADES approach. However, the MCNP full physics treatment is the one which should be used to study scatter in general.

Given the two HADES approximations discussed above, we see fairly good verification of the HADES simulations by MCNP. These results are heartening because HADES appears to be performing the scatter calculation algorithm specified in Section 3. Future work will focus on removing these approximations and more verification and validation tests.

\section{Physics Implications}

We have used the MCNP studies to verify treatment of single Compton scatter. However, it is also useful to use MCNP to study the full problem of scatter as described in Section 2. In order to study scatter we use all three types of MCNP simulations. The full simulation is used to show the effect of all X-ray scatter processes (except coherent) and the effect of electron/positron production, secondary bremsstrahlung and positron annihilation. The simulation with material densities set to $0.001 \mathrm{~g} / \mathrm{cc}$ provides estimates for the source intensity with no attenuation. The case with electron-positron transport of and all processes but Compton scatter off allow the contributions due to multiple Compton scatters to be probed.

In Figure 5a and Figure 5b we show these calculations plotted for $6 \mathrm{MeV}$ and $9 \mathrm{MeV}$, respectively, for the $9 \mathrm{~cm}$ detector. All scatter, if summed up, accounts for $0.02 \%(6 \mathrm{MeV})$ and $0.04 \%(9 \mathrm{MeV})$ of the direct signal, neglecting the top bin, for the $9 \mathrm{~cm}$ detector. It can be seen that, for high energies, close to the source energy, the dominant contributor to scatter is single Compton scatter, which is roughly $40 \%$ of the full scatter signal for both sources . However, below these few top bins, the scatter is dominated by double Compton scatter, which accounts for $20 \%(6 \mathrm{MeV})$ and $9 \%(9 \mathrm{MeV})$ of the total scatter flux. Triple Compton scatter accounts for $5 \%(6 \mathrm{MeV})$ and $2 \%(9 \mathrm{MeV})$ of the total scatter flux. The rest of the scatter comes from processes which produce photons as a result of the scatter and transport of electrons and positrons. Overall, these processes account for $34 \%(6 \mathrm{MeV})$ and $46 \%(9 \mathrm{MeV})$ of the total 
Scatter signal.

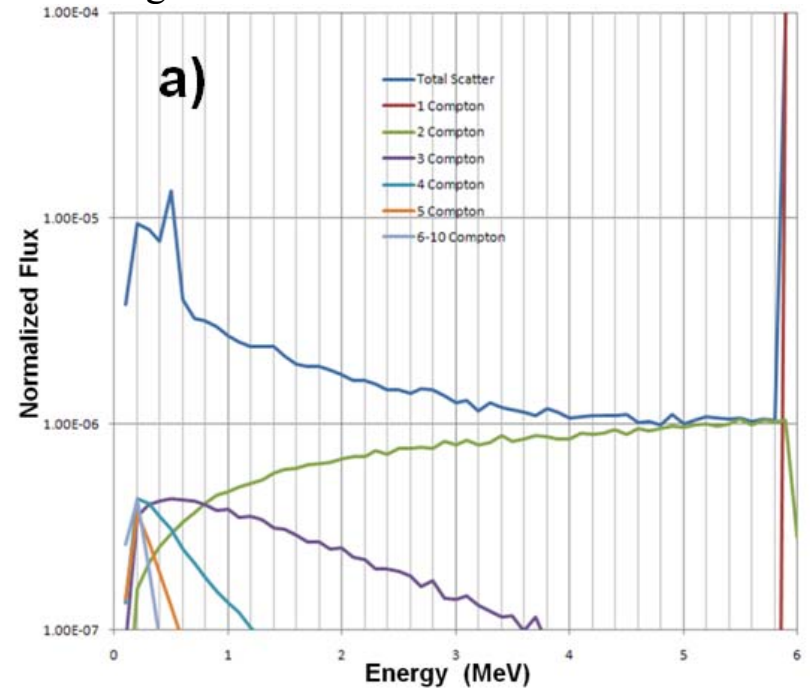

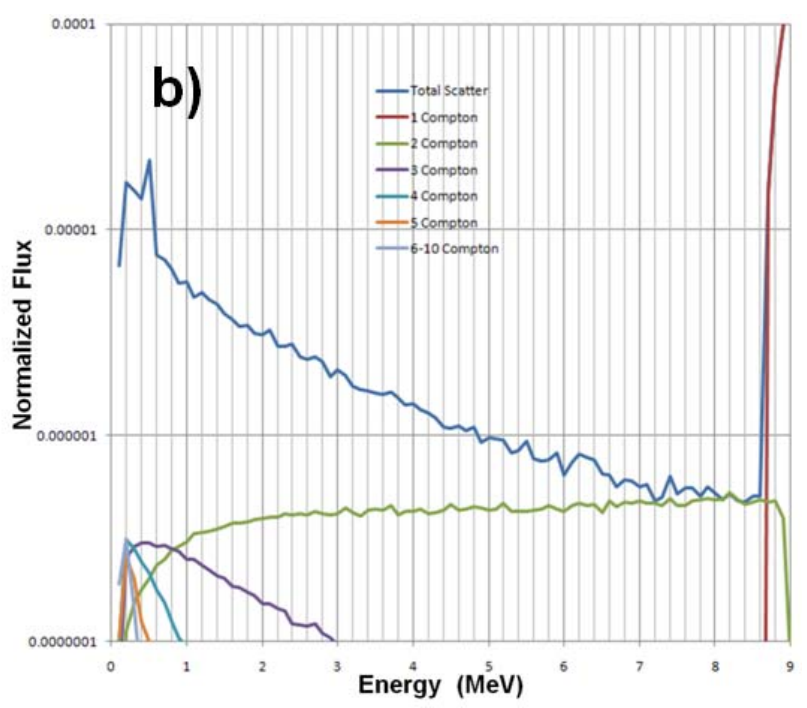

Figure 5: Contributions to scatter for the a) $6 \mathrm{MeV}$ and b) $9 \mathrm{MeV}$ source for the $9 \mathrm{~cm}$ detector.

Similar results are displayed in Figure 6a and Figure 6b, in which similar plots are made for the detector at $0 \mathrm{~cm}$. The main difference between the two detectors is that the $9 \mathrm{~cm}$ detector sees a little more scatter in general. Also, the $0 \mathrm{~cm}$ detector sees very little single Compton scatter, since the angles in the problem put most of the single Compton scatter back into the source energy bin.
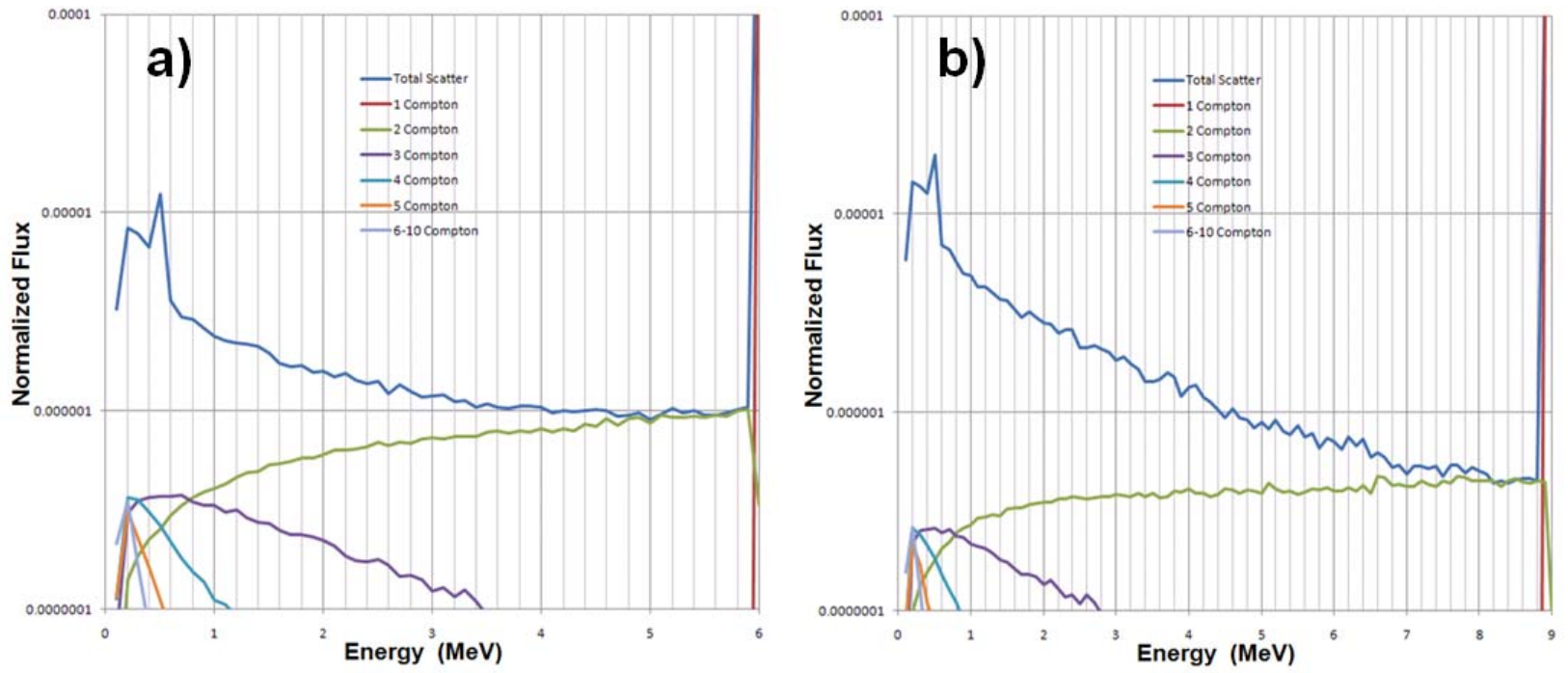

Figure 6: Contributions to scatter for the a) $6 \mathrm{MeV}$ and b) $9 \mathrm{MeV}$ source for the $0 \mathrm{~cm}$ detector.

A major conclusion to be drawn from these simulations is that single Compton scatter is an important component of scatter, but not the dominant one. Even in the case of a full source spectrum, the single Compton scatter would be the largest individual contributor at energies nearest the source bins, but ever more scatter at lower energies would swamp the single Compton contribution. Thus, other processes also need to be considered when including scatter in radiographic simulations. 


\section{Path Forward}

Now that we have achieved some level of verification, HADES needs to be refined in its treatment. First, the approximations described above (detector norms and proper energy placement of scattered radiation) need to be done. A next refinement will be to include coherent scatter cross sections into the code as a second process. This process has a stronger angular dependence than Compton scatter (within roughly $10^{\circ}$ of forward peaking). This inclusion is likely to help explain scatters which are strongly correlated with the direct signal, seen in lower energy radiography.

We have seen in the previous section that a great deal of scatter comes from two and three Compton scatters, in addition to secondary bremsstrahlung and pair production. It is possible to derive and implement approximate differential cross sections for pair production and secondary bremsstrahlung, but the calculations would be challenging. A major cause of the difficulty is the multiple Coulomb scattering which electrons (and positrons) experience as they propagate through materials. Deriving differential cross sections for multiple Compton scattering would be even more challenging.

A better approach to this challenge would be use Monte Carlo to build effective differential cross sections for these processes. In fact, if one takes this approach, one could just lump all of the processes together (including Compton scatter) and use Monte Carlo to build an effective total differential cross section. One may ask what is gained from such an approach? As seen above, the HADES simulations were much faster than the MCNP simulations, albeit for a simpler calculation. Using this effective approach would require running the Monte Carlo a great deal, once, to build a library for HADES, after which every HADES simulation would be just as fast as the current approach. This approach is very similar to the way in which HADES uses Monte Carlo generated source and detector libraries at the start and end of every simulation.

Let us give a brief summary of how such a project could be carried out. A Monte Carlo model of a monochromatic photon source, emanating from one point in only one direction would be built. A series of Monte Carlo simulations with this point source, surrounded by spheres of varying elements and radii, would be performed with all transport processes on and $180 \mathrm{f5}$ spectral detectors. These detectors would be placed at the same radius at points $1^{\circ}, 2^{\circ}, \ldots 180^{\circ}$ from the source direction, so that the scatter into this angle could be calculated. The density of the material would be set at the standard density for that material, although this quantity would need to be divided out so that small density changes could be accounted for when used by HADES.

This approach would be studied for several important elements: $\mathrm{N}_{2}, \mathrm{C}, \mathrm{Al}, \mathrm{Fe}, \mathrm{W}, \mathrm{Pb}$ and U. The ranges of incident energy and material radius would be varied to see how the emitted scatter varies with these properties. Also, the convergence properties of the detectors would need to be studied in order to assess how much MCNP time is needed for each run. These studies also would allow observation of competing processes, such as self-shielding and varying electron scatter lengths, to be varied. One important issue to resolve with these studies is to make sure that HADES does not double count attenuation of the scatter as it is produced in the object. In addition, these studies might illustrate how large the stepping should be in HADES.

\section{Conclusions}

In this report, we have explained and demonstrated how single Compton scatter has been implemented in HADES. In Section 2 we delineated the many kinds of scatter which can 
contribute to X-ray radiography. We distinguish these kinds of scatter from single Compton scatter. In Section 3 we explained how single Compton scatter is implemented in HADES. This implementation is a major expansion in HADES' capability, which opens the possibility of many other applications.

In Section 4 we demonstrated HADES scatter calculation for two objects in a geometry similar to what is seen in CAARS scanners. In this case, we used a two dimensional detector instead of the linear detectors, in order to increase the amount of scatter produced. It was seen that the nature of the calculated scatter was consistent with the angles involved in these case. The HADES calculations were then compared to MCNP simulations. Verification was obtained, to the extent that the calculations were comparable. Two HADES approximations were found which need to be refined in future work. The MCNP calculations also showed that other scatter processes such as secondary bremsstrahlung, two and three Compton scatter and pair production are major contributors to the total scatter.

In Section 5 we discussed how the new scatter information infrastructure in HADES could be used. Coherent scatter could be included in simulations relatively easily, by including the coherent scatter cross section, in a manner analogous to the way in which single Compton scatter has been treated. The rest of the section described a strategy for building Monte Carlo libraries to include all scatter processes in HADES.

\section{Acknowledgements}

Sean Walston wrote the code which models the Klein-Nishina cross section in HADES. We thank Alexis Schach von Wittenau, Doug Wright, Harry Martz, Michael Curtin, Gene Luong, Celestino Abrego, and Doug Wright for many useful discussions about scatter. HADES work has been funded under the auspices of the U.S. Department of Energy by Lawrence Livermore National Laboratory in part under Contract W-7405-Eng-48 and in part under Contract DE-AC52-07NA27344. This new work on HADES was funded by the Department of Homeland Security under interagency agreement HSHQDC-08-X-00388.

\section{Bibliography} 71 (1), 3-10.

Baldwin, G., \& Klaiber, G. (1947). Photo-fission in Heavy Elements. Physical Review,

BRL-CAD Open Source Project. (2010). BRL-CAD: Open Source Solid Modeling. Retrieved from http://brlcad.org/

Cullen, D., Perkins, S., \& Rathkopf, J. (1990). The 1989 Livermore Evaluated Photon

Data Library (EPDL). UCRL-ID-103424.

L106.

Dicke, R. (1968). Scatter-Hole Cameras for X-rays and Gamma Rays. Ap. J. , L101-

Evans, R. D. (1985). The Atomic Nucleus. Krieger Publishing Company.

Groh, G., Hayat, G., \& Stroke, G. (1972). X-ray and Gamm Ray Imaging with Multiple-

Pinhole Cameras Using a Posteriori Image Synthesis. Applied Optics , 931-933.

Holt, S. (1976). Temporal X-Ray Astronomy with a Pinhole Camera. Astrophysics and Space Science, 123-141.

Hubbell, J. (2006). Electron-positron pair production by photons: A historical overview. Radiation Physics and Chemistry , 75 (6), 614-623. 
Key, M., Lewis, C., Lunney, J., Moore, A., Hall, T., \& Evans, R. (1978). Pulsed-X-Ray Shadowgraphy of Dense, Cool, Laser-Imploded Plasma. Phys. Rev. Lett. , 41, 1467-1470.

Klein, O., \& Nishina, Y. (1929). Über die Streuung von Strahlung durch freie Elektronen nach der neuen relativistischen Quantendynamik von Dirac. Z. F. Phys. , 52, 853-869.

McDonald, K. T. (1998). Higher-Order QED Effects and Nonlinear QED. XVIII Physics in Collision. Frascati.

Miyanaga, M., Kato, Y., \& Yamanaka, C. (1983). Point-source x-ray backlighting for high-density plasma diagnostics. Appl. Phys. Lett. , 42, 160-162.

Moses, W., Gayshan, V., \& Gektin, A. (2006). The Evolution of SPECT from Anger to Today and Beyond. Radiation Detectors for Medical Applications (pp. 37-80). NATO Security through Science Series.

Rybicki, G. B., \& Lightman, A. P. (1979). Radiative Processes in Astrophysics. John Wiley \& Sons, Inc.

Schiff, L. (1946). Resonance Fluorescence of Nuclei. Physical Review , 70, 761-762.

Wattenberg, A. (1947). Photo-Neutron Sources and the Energy of the Photo-Neutrons. Physical Review , 71 (8), 497-507.

X-5 Monte Carlo Team, /. /. (2005 Revision). MCNP - A General Monte Carlo NParticle Transport Code, Version 5. Los Alamos National Laboratory. LA-UR-03-1987. 